\title{
Reduction of body fat and improved lipid profile associated with daily consumption of a Puer tea extract in a hyperlipidemic population: a randomized placebo-controlled trial
}

\author{
This article was published in the following Dove Press journal: \\ Clinical Interventions in Aging \\ 24 March 2016 \\ Number of times this article has been viewed
}

\author{
Gitte S Jensen' \\ Joni L Beaman' \\ Yi $\mathrm{He}^{2}$ \\ Zhixin Guo 2 \\ Henry Sun ${ }^{3}$ \\ 'NIS Labs, Klamath Falls, OR, USA; \\ ${ }^{2}$ Modern TCM Research Center, Tasly \\ Academy, Tianjin, People's Republic \\ of China; ${ }^{3}$ Tasly Pharmaceuticals Inc, \\ Rockville, MD, USA
}

\begin{abstract}
Objective: The goal for this study was to evaluate the effects of daily consumption of Puer tea extract (PTE) on body weight, body-fat composition, and lipid profile in a non-Asian population in the absence of dietary restrictions.
\end{abstract}

Materials and methods: A randomized, double-blind, placebo-controlled study design was used. A total of 59 overweight or mildly obese subjects were enrolled upon screening to confirm fasting cholesterol level at or above $220 \mathrm{mg} / \mathrm{dL}$ (5.7 mmol/dL). After giving informed consent, subjects were randomized to consume PTE ( $3 \mathrm{~g} /$ day) or placebo for 20 weeks. At baseline and at 4-week intervals, blood lipids, C-reactive protein, and fasting blood glucose were evaluated. A dual-energy X-ray absorptiometry scan was performed at baseline and at study exit to evaluate changes to body composition. Appetite and physical and mental energy were scored at each visit using visual analog scales (0-100).

Results: Consumption of PTE was associated with statistically significant weight loss when compared to placebo $(P<0.05)$. Fat loss was seen for arms, legs, and the gynoid region (hip/belly), as well as for total fat mass. The fat reduction reached significance on within-group analysis, but did not reach between-group significance. Consumption of PTE was associated with improvements to lipid profile, including a mild reduction in cholesterol and the cholesterol:high-density lipoprotein ratio after only 4 weeks, as well as a reduction in triglycerides and very small-density lipoproteins, where average blood levels reached normal range at 8 weeks and remained within normal range for the duration of the study $(P<0.08)$. No significant changes between the PTE group and the placebo group were seen for fasting glucose or $\mathrm{C}$-reactive protein. A transient reduction in appetite was seen in the PTE group when compared to placebo $(P<0.1)$.

Conclusion: The results from this clinical study showed that the daily consumption of PTE was associated with significant weight loss, reduced body mass index, and an improved lipid profile. Keywords: body mass index, DEXA, cholesterol, triglycerides

\section{Introduction}

Obesity has become a critical worldwide health concern in the past few decades, as the worldwide prevalence of obesity has more than doubled over the past 35 years. The World Health Organization estimated there were more than 1.9 billion overweight adults 18 years and older in the year 2014. Of these, over 600 million were obese. ${ }^{1}$ Obesity is a major risk factor for multiple health problems, including type 2 diabetes, ${ }^{2}$ coronary heart disease, ${ }^{3}$ sleep apnea, ${ }^{4}$ osteoarthritis,${ }^{5}$ and certain forms of cancer, ${ }^{6-8}$ as well as increased risk of death from such diseases. ${ }^{9}$ Weight loss can help reduce
Correspondence: Gitte S Jensen NIS Labs, 1437 Esplanade Avenue, Klamath Falls, OR 9760I, USA

$\mathrm{Tel}+\mathrm{I} 54 \mathrm{I} 88401 \mathrm{I} 2$

Fax +I $40344 \mid 5236$

Email gitte@nislabs.com

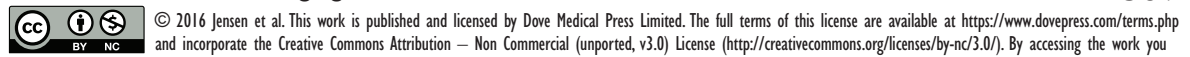
hereby accept the Terms. Non-commercial uses of the work are permitted without any further permission from Dove Medical Press Limited, provided the work is properly attributed. For permission for commercial use of this work, please see paragraphs 4.2 and 5 of our Terms (https://www.dovepress.com/terms.php). 
blood pressure and blood cholesterol, and improve glucose tolerance in people prone to diabetes. ${ }^{10}$

Although robust weight loss may be ideal for obese people, even a modest reduction in weight can have clinically meaningful and significant health benefits. For the management of obesity, behavior-based (diet and lifestyle) treatment programs have been shown to improve weight-loss results as the most commonly used treatment. ${ }^{11}$ Beverages, such as teas brewed from the leaves of Camellia sinensis, are easily incorporated into a daily routine. Various types of tea are categorized based on the degree of fermentation during manufacturing, each of the four types with different chemical composition and health benefits: nonfermented (green) tea, partially fermented (oolong) tea, fully fermented (black) tea, and postfermented tea (Puer). ${ }^{12-15}$

Puer tea originated in Yunnan Province in imperial China, and has long been consumed as one of the most popular beverages in Asia. ${ }^{16}$ Historically, Puer tea was used as a medicinal drink in the treatment of a variety of illnesses, such as common colds, flatulence, poor digestion, and onset of dysentery. ${ }^{17}$ In the past few decades, the unique effects of Puer tea have been extensively studied, and results indicate that Puer tea possesses a broad range of health-promoting effects, including hypoglycemic effects ${ }^{18,19}$ and improvement of type II diabetes, as well as inhibition of the progression of diabetic complications. ${ }^{20}$

Of pertinence to the study reported here, studies in rodents have shown that consumption of Puer tea can decrease weight gain ${ }^{21,22}$ and improve hyperlipidemia conditions. ${ }^{23-25}$ The beneficial properties of Puer tea may in part be attributable to the bioactive components theabrownin, ${ }^{26}$ theaflavins, ${ }^{12}$ and complex polysaccharides. ${ }^{27}$ The statin compound lovastatin has been identified in Puer tea, ${ }^{28}$ and lovastatin levels increase with fermentation of tea leaves. ${ }^{29,30}$ However, the levels of statins that can be found in some sources of Puer tea are several hundredfold lower than a low dose of statin delivered as a drug for hyperlipidemia, ${ }^{29,31}$ and thus quite unlikely to contribute significantly to the overall effects of Puer tea consumption.

Despite the large volume of studies of Puer tea in rodents, only a few clinical studies have been conducted to investigate the health-promoting effect of Puer tea in humans. A 3-month double-blind randomized study was performed in borderline hypercholesterolemic human subjects in a Japanese population. The result showed significant improvements in lipid profile. ${ }^{32}$ The antiobesity effect of Puer tea was documented in overweight Japanese adults (body mass index [BMI] $25-30 \mathrm{~kg} / \mathrm{m}^{2}$ ), where intake of Puer tea was associated with reduction of body weight, mean waist circumference, BMI, and visceral fat. ${ }^{33}$ Previous publications on human clinical trials documenting the effects of Puer tea were conducted in populations of Asian ethnicity, diet, and lifestyle. The study reported here aimed at evaluating changes in weight, fat mass, and lipid profile in hyperlipidemic subjects in a North American population.

\section{Materials and methods Study design}

A randomized, double-blind, placebo-controlled study design was used for this clinical study, with a duration of 20 weeks and visits every 4 weeks. A total of 59 overweight or mildly obese adult men and women were enrolled in the study after screening for cholesterol level at $220 \mathrm{mg} / \mathrm{dL}$ or higher and signing written informed consent. This study was approved by the ethical committee of Sky Lakes Medical Center. The study population comprised of 58 Caucasians and one Hispanic. An even sex distribution was not a criteria for the study, and a female:male ratio of 2:1 reflected the higher interest and availability of women to participate in this type of clinical trial. Inclusion criteria were 35-75 years of age, BMI 25-35 kg/m²; waist circumference higher than $80 \mathrm{~cm}$ for women and higher than $96 \mathrm{~cm}$ for men, and fasting cholesterol level of $220 \mathrm{mg} / \mathrm{dL}$ or higher. Exclusion criteria were: treated with statin hyperlipidemia medication for the past month; treated with other medication specifically for hyperlipidemia; daily alcohol use of four or more standard units/day (or 28 or more per week); screening blood results of complete blood count and liver and renal function judged to be of clinical significance; currently treated with insulin (other forms of diabetic medication were allowed); pregnancy (a pregnancy test was provided at screening for pre- and perimenopausal women); unwilling to maintain a constant regimen of medication and supplements for the duration of the study (based on their habitual consumption during the past 2 months prior to entering this study), with the exception of changes to medication needed for the optimal care of each person; blood donation during the 2 weeks prior to study start; participation in other clinical trials during the last month; and food allergies or sensitivities related to the test product.

\section{Consumables}

The Puer tea and placebo powders were prepared in a servingsize sachet, boxed, and labeled with instructions at Tasly Pharmaceuticals Inc. The placebo powder was prepared from dextrin produced from cornstarch. Packages of a 4-week (56 servings for 28 days) supply were prepared. Subjects 
were randomized to receive either placebo or Puer tea for the duration of the study. At the baseline visit and visits at 4, 8, 12, and 16 weeks, subjects were provided with a 4-week supply of their assigned consumable. Subjects were instructed to consume two $1.5 \mathrm{~g}$ portions daily with meals, with instructions to dissolve the content of one sachet in approximately one cup warm water $(250 \mathrm{~mL})$ immediately before consuming. Subjects were allowed to add a small amount of milk and/or sugar. Adherence to the study protocol was evaluated at each visit by documentation of the returned product, an interview with the study coordinator, and review of the diary.

\section{Blood pressure measurements}

Blood pressure was recorded at each visit using an Omron BP 742 monitor, after the subject had sat quietly for 3 minutes.

\section{Blood testing}

Blood was drawn using venipuncture at each visit and tested for lipid profile, C-reactive protein (CRP), and fasting blood glucose.

\section{Body measurements}

Body height was measured at the study start. Weight measurements were performed at each visit using a mechanical scale. A fabric tape was used to perform measurements of the waist, hip, thigh, and upper arms. The waist measurement was performed at the smallest area of the trunk for women, and at the navel line for women without an apparent waist. The waist measurement for men was always taken at the navel line. Subjects were instructed to relax and not flex the abdominal muscles during the waist measurement. Upperarm measurements were performed on the dominant side of the body, with the arm held outstretched and parallel to the floor while the largest point above the elbow was measured. Hip measurements were performed around the largest circumference of the body below the waist and above the thigh. Thigh measurements were performed on the dominant side of the body, at the largest point above the knee, with the subject standing with the legs a shoulder width apart.

\section{Dual energy X-ray absorptiometry}

Body-fat composition was performed by whole-body dualenergy X-ray absorptiometry (DEXA) scanning, using a Lunar DEXA scanner (Prodigy series X-ray; GE Healthcare, Little Chalfont, UK). A quality-assurance calibration scan was performed once a day prior to scanning the subjects. Subjects were positioned in the center of the table with their arms separated from the sides of the body and their hands placed with the palms against their thighs and the elbows tucked into their sides. Their knees and ankles were strapped into place. Their head was adjusted so the midline of the table was aligned with the middle of the head, and the subject's legs were adjusted so the midline of the table was between the legs. The scan lasted 6-11 minutes, depending on the size of the volunteer. If the volunteer moved during the scan, the scan was aborted, the person was then repositioned, and the scan was restarted. The resulting data from the DEXA scan were analyzed using Encore 2011 software, where regional analysis includes lean and fat mass for arms, legs, trunk, android (belt area), gynoid (hip area), and total body.

\section{Questionnaire-based data collection}

At each visit, questions pertaining to appetite and satiety were scored using unmarked visual analog scales.

\section{Statistical analysis}

Average and standard deviation for each data set were calculated using Microsoft Excel. Statistical analysis of clinical data was performed as between-group comparison of the group averages for each time point in the study using the two-tailed, unpaired $t$-test. The statistical significance of changes from baseline to later assessments was evaluated by between-treatment analysis using within-subject analysis and the two-tailed paired $t$-test. Statistical significance was indicated by $P<0.05$, and a high level of significance was indicated by $P<0.01$.

\section{Results}

\section{Study enrollment, compliance, and completion}

In order to complete the enrollment for this study, a total of 171 people were screened, and 112 failed screening: 85 failed due to cholesterol below $220 \mathrm{mg} / \mathrm{dL}$, one was excluded because of extremely high triglycerides (over $500 \mathrm{mg} / \mathrm{dL}$ ), eight were excluded because of their BMI, and the remaining subjects failed screening for other reasons.

Of the 59 people enrolled, 49 completed the 20 -week participation (Figure 1). Four people in the placebo group and one in the Puer tea extract (PTE) group discontinued study participation for various medical reasons, including change in medication and health status. An additional five people discontinued participation at various times during the 20-week study, due to practical issues (moving, traveling) or loss of interest. One female subject who completed the study was removed from analysis due to noncompliance in 


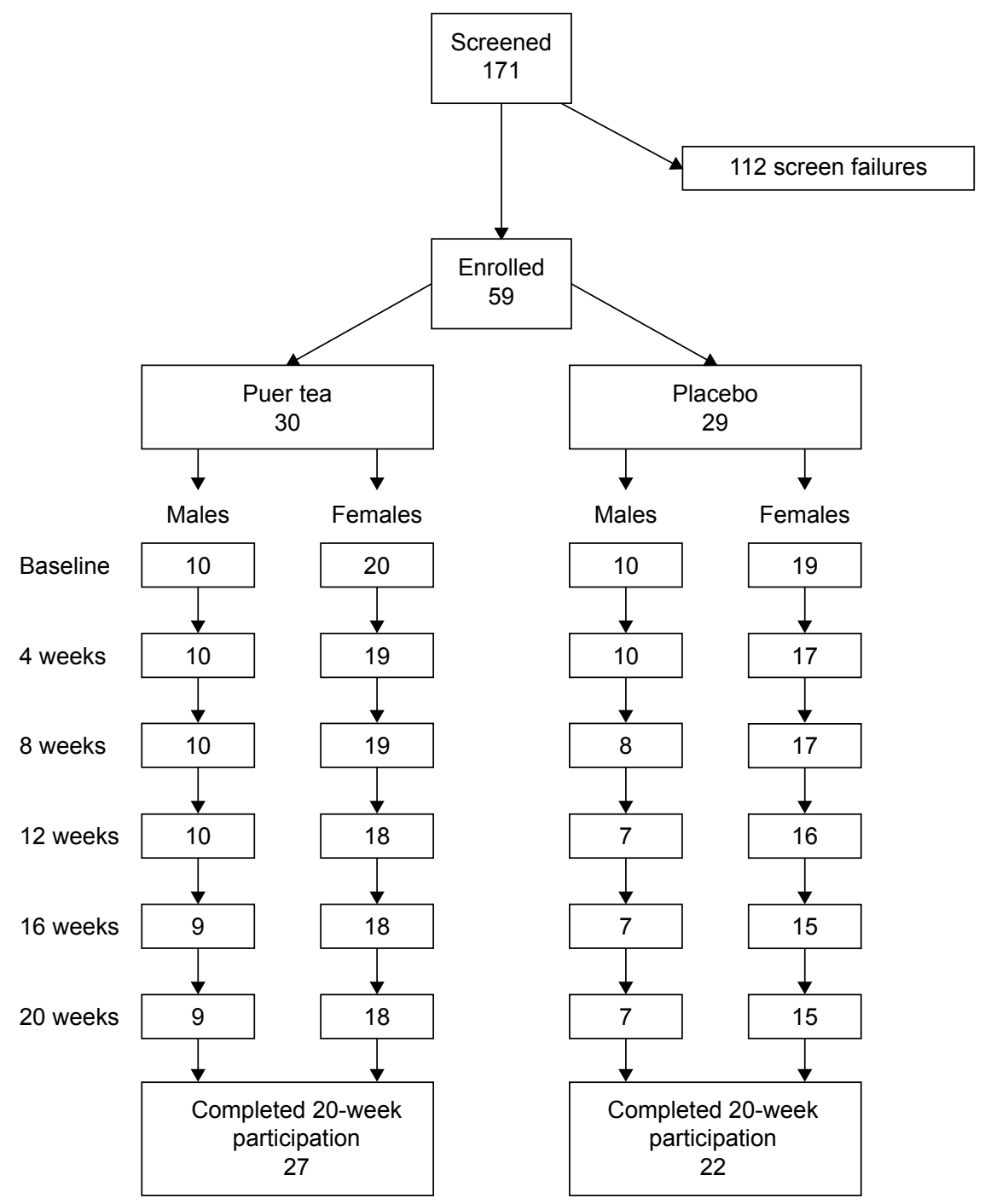

Figure I Consort flowchart.

Note: Chart shows screening and randomization, as well as the number of people completing each 4-week phase of the 20-week study on Puer tea extract.

terms of maintaining a constant diet and lifestyle during the study, which was manifested by a substantial weight gain (29\% increase in body weight during the study, compared to her body weight at baseline).

There were no statistically significant differences between age, BMI, or physical measurements between the subjects randomized to placebo versus PTE for either the female or male subject group (Table 1).

\section{Body mass index, body measurements, and body-fat composition}

A statistically significant reduction in BMI was seen at 20 weeks for the PTE group when compared to the placebo group $(P<0.05)$ (Figure 2$)$. The average reduction in body weight in the PTE group was small $(1 \mathrm{~kg})$, but highly significant $(P<0.01)$.
Body measurements showed a decrease in hip and waist measurements in the PTE group, but the changes did not reach statistical significance (data not shown). Body measurements showed an increase in thigh circumference at study exit for the subjects in the PTE group, which associated with the fat loss for the same region suggested an increase in muscle mass for that region.

The weight loss corresponded to a reduction in total body fat at 20 weeks for the PTE group when compared to the placebo group (Figure 3A). This change was not statistically significant between the two groups, but the change was significant within the PTE group $(P<0.05)$.

The loss of body fat was seen in the trunk, arm, and leg regions (Figure 3B-D). Regional analysis of trunk fat showed that the fat loss was less obvious for the android region (Figure $3 \mathrm{E}$ ), but was predominant for the gynoid 
Table I Characteristics of study population

\begin{tabular}{llll}
\hline & Placebo & Puer tea extract & P-value* \\
\hline Females & 19 & 20 & \\
Average age, years & $55 \pm 10$ & $57 \pm 9$ & 0.589 \\
Age range, years & $38.1-70.1$ & $38.7-72.3$ & \\
BMI average & $30.7 \pm 4.5$ & $29.3 \pm 3.2$ & 0.257 \\
BMI range & $25.2-40$ & $25.1-35.2$ & \\
Height average, cm & $163 \pm 6$ & $163 \pm 5$ & 0.990 \\
Height range, cm & $147.3-171.5$ & $153.7-172.7$ & \\
Weight average, kg & $82 \pm 15$ & $78 \pm 10$ & 0.339 \\
Weight range, kg & $59.1-114.1$ & $65.9-98.2$ & \\
Males & 10 & 10 & \\
Average age, years & $55 \pm 11$ & $52 \pm 8$ & 0.616 \\
Age range, years & $42-73.4$ & $39.2-65.9$ & \\
BMI average & $29 \pm 3$ & $31 \pm 4$ & 0.269 \\
BMI range & $25.8-36.7$ & $26.4-39.5$ & \\
Height average, cm & $177 \pm 7$ & $177 \pm 7$ & 0.935 \\
Height range, cm & $169.5-190.5$ & $160-184.8$ & \\
Weight average, kg & $92 \pm 12$ & $98 \pm 15$ & 0.369 \\
Weight range, $\mathrm{kg}$ & $76.8-113.6$ & $76.1-126.8$ & \\
\hline
\end{tabular}

Notes: *There were no statistically significant differences between age and physical measurements between the subjects randomized to placebo versus Puer tea for either female or male subject group. Data presented as $\mathrm{n}$ or mean \pm standard deviation. Abbreviation: BMI, body mass index.

(hip) region (Figure 3F). There was no statistical difference in gynoid fat between the groups at study exit, but when the data within the PTE group were analyzed, the fat loss in the gynoid region was statistically significant $(P<0.05)$. There were no significant differences in lean or bone mass between the two groups (data not shown).

Self-reported appetite showed a high degree of consistency in the PTE group, whereas the placebo group showed

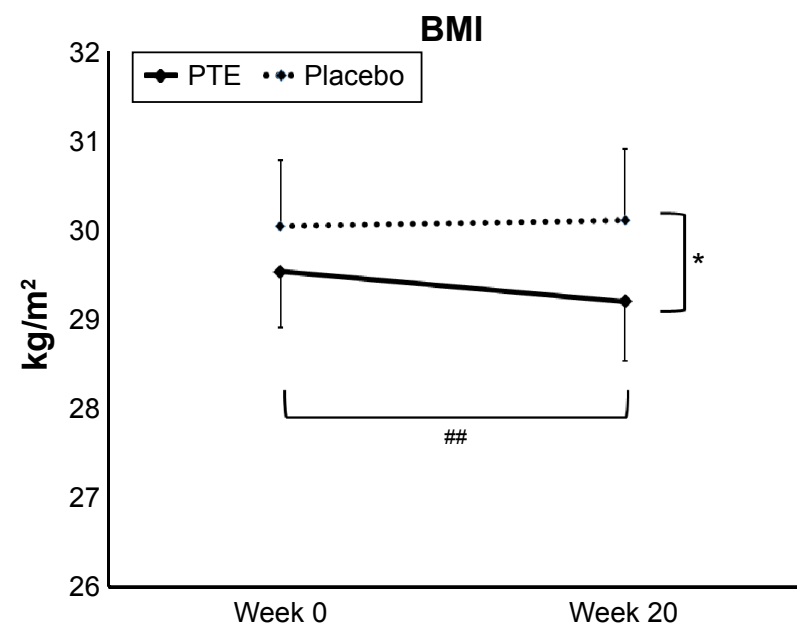

Figure 2 Body mass index (BMI).

Notes: Shown as the group averages \pm standard error of mean for the Puer tea extract (PTE) group (solid lines) and the placebo group (dashed lines). There was a significant difference in BMI between the two groups at week 20. The BMI reduction within the PTE group over the course of the 20 -week study was highly significant. $* P<0.05, \ldots P<0.01$. variations across the 20 weeks. Lower appetite was seen for weeks 4-16 in the PTE group when compared to placebo, reaching a statistical trend at week $8(P<0.1)$. No other significant differences were seen.

\section{Lipid profile}

There was no significant difference in the cholesterol levels between the two groups at any time during the study (Figure 4A). However, within the PTE group, data showed that consumption of PTE was associated with a significant reduction in cholesterol levels, starting at week 4 and remaining significantly lower than baseline throughout the study. In contrast, the changes within the placebo group showed only a transient lowering of cholesterol levels at weeks 4 and 8, likely due to a transient increase in awareness of diet and lifestyle in the early phase of study participation, and returning almost to baseline levels at weeks 12 , 16 , and 20 .

There was no significant difference in high-density lipoprotein (HDL) levels between the two groups at any time during the study (Figure 4B). Under within-group analysis, consumption of placebo was associated with a statistical trend for lower HDL levels at week 8.

There was no significant difference in cholesterol:HDL levels between the two groups at any time during the study (data not shown). However, under within-group analysis, consumption of PTE was associated with statistical trends for lowering the cholesterol:HDL levels at weeks 4 and 20, and with statistical significance at week 16 .

Low-density lipoprotein levels were not significantly affected by PTE consumption in this study population (Figure 4C). A transient reduction in low-density lipoprotein levels was seen in both the PTE and placebo groups, suggesting that the awareness of being in a weight-management study with a focus on cholesterol/weight management may have affected fat intake.

When compared to the placebo group at week 20 , reduced triglyceride levels in the PTE group showed a trend $(P<0.1)$ (Figure 4D). The average percentage change showed a trend for reduced triglyceride levels in the PTE group when compared to the placebo group at week 8. Under within-group analysis, consumption of PTE was associated with a significant reduction in triglyceride levels at week 8 and continued to decrease throughout the 20 -week study, with the exception of a mild transient increase at week 12. A transient increase in triglyceride levels was seen in both groups at week 12 ; this transient increase reached a statistical trend for the placebo group, but was not significant in the PTE group. 

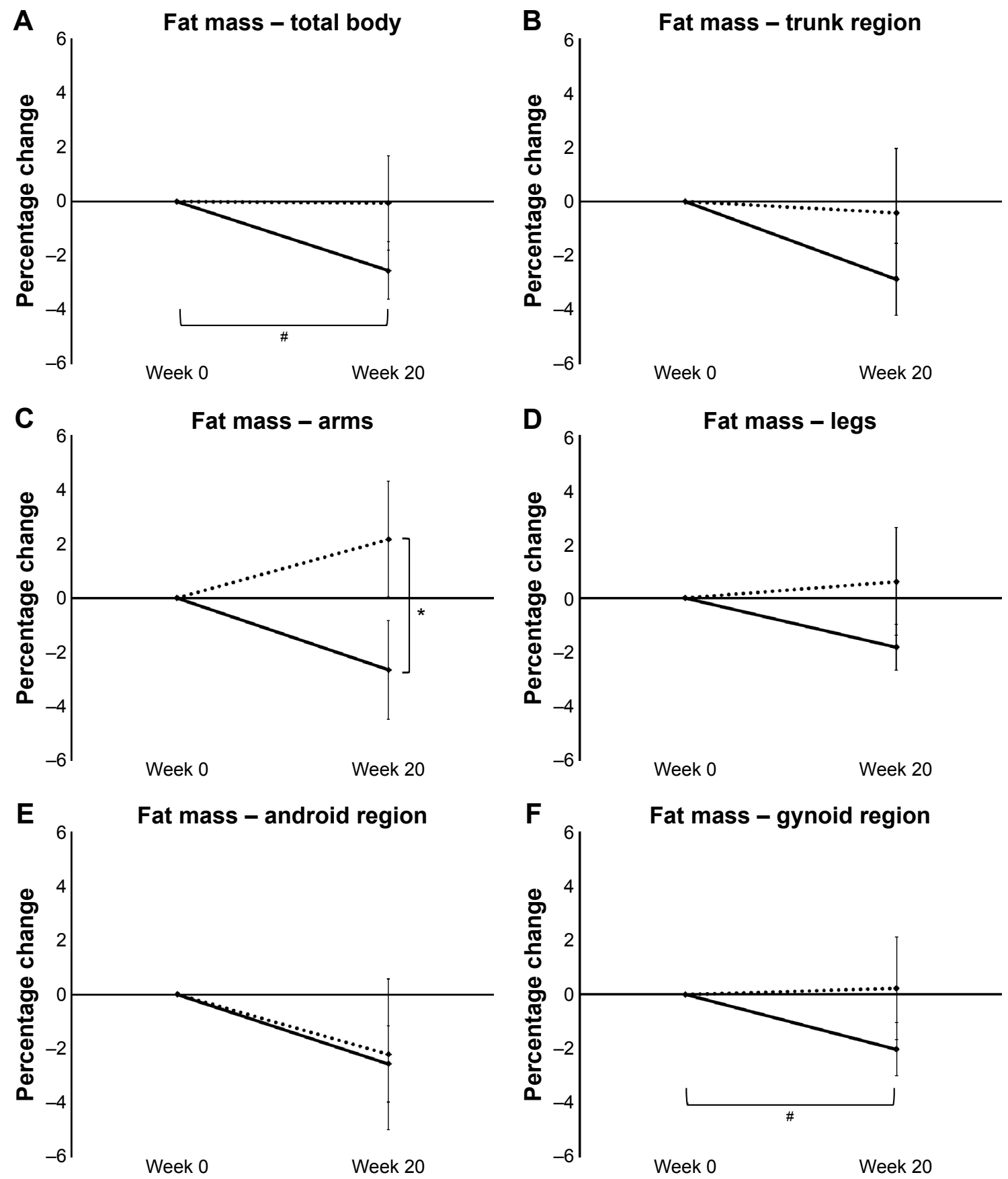

PTE .»P Placebo

Figure 3 Changes in total body fat and regional fat composition.

Notes: Shown as the group averages of the individual percentage changes \pm standard error of mean for the Puer tea extract (PTE) group (solid lines) and the placebo group (dashed lines). There were no significant differences between the two groups at week 20; however, the between-group difference in fat loss in the arms showed a statistical trend. Within the group consuming PTE, a significant loss of total body fat was seen. The total fat loss was associated with regional fat loss in the trunk, arms, thighs, and gynoid (belly/hip) regions. The fat loss in the gynoid region within the PTE group was statistically significant. $* P<0.1,{ }^{\sharp} P<0.05$.

\section{Blood pressure}

There was no change in blood pressure in either group, despite the content of stimulants in PTE. There were no statistically significant differences between average systolic or diastolic blood pressure at baseline or during the study in either the female or male subpopulation (Table 2).

\section{C-reactive protein}

Blood samples were tested for CRP levels at each visit. At baseline, the average CRP level in the PTE group was $0.25 \pm 0.04 \mathrm{mg} / \mathrm{dL}$, and in the placebo group was $0.22 \pm 0.04 \mathrm{mg} / \mathrm{dL}$. Over the course of the 20-week study, average high-sensitivity CRP levels remained well within the normal range (below $3.0 \mathrm{mg} / \mathrm{dL}$ ), and 

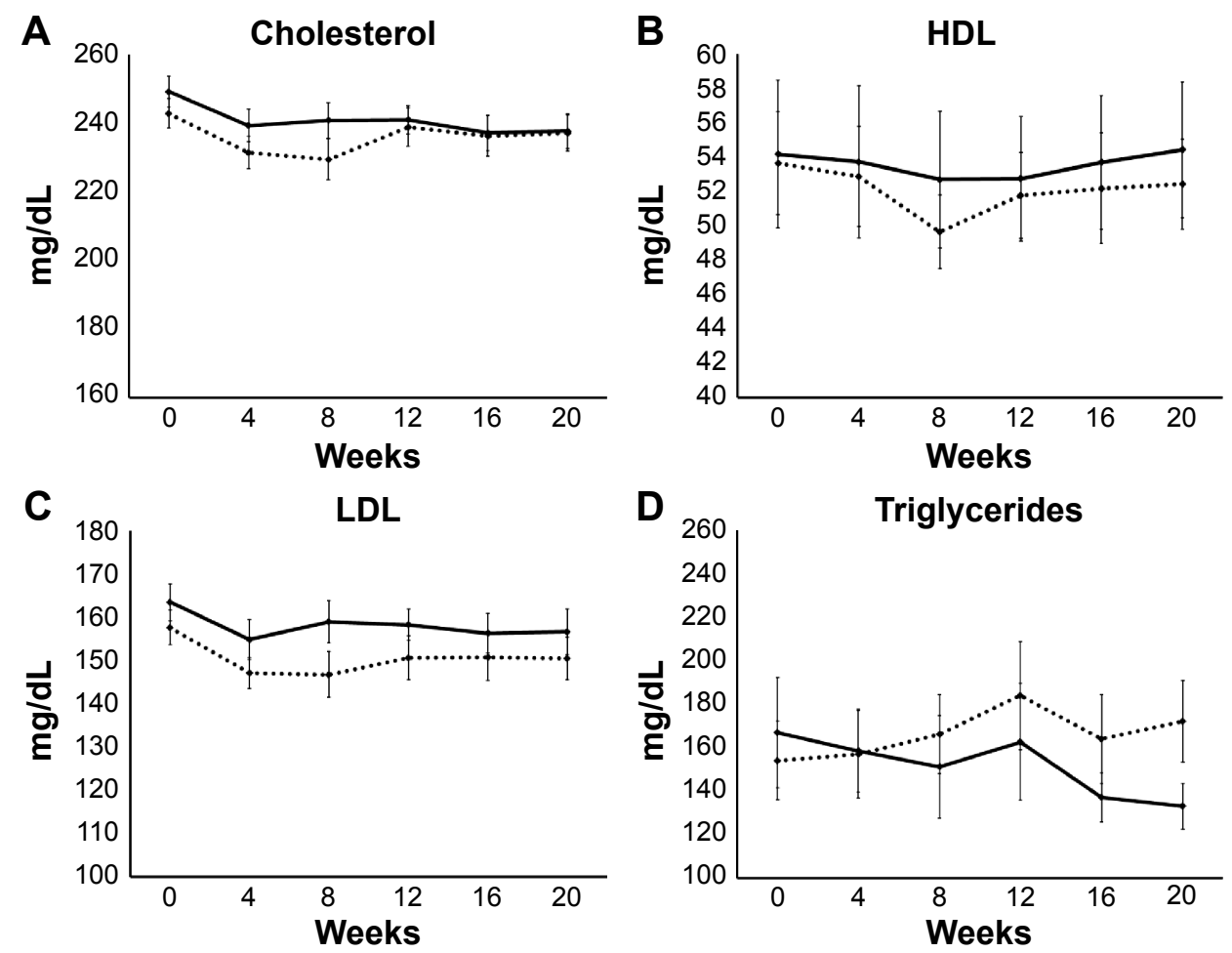

Figure 4 Lipid data are shown as group averages \pm standard error of mean for the Puer tea extract (PTE) group (solid lines) and the placebo group (dashed lines). Notes: There were no significant differences between the PTE and placebo results for cholesterol (A), HDL (B), or LDL (C). Within the PTE group, a significant reduction in cholesterol levels was seen already at week 4, and remained statistically significantly lower than baseline throughout the study. Triglyceride levels showed a reduction in the PTE group that was not statistically significant, but was clinically important (D): the group average in the PTE group went down into the normal range (below I50 mg/dL) after 8 weeks, and remained low throughout the remainder of the study. After 20 weeks, there was a $20 \%$ reduction in the average triglyceride levels within the PTE group $(P<0.08)$.

Abbreviations: HDL, high-density lipoprotein; LDL, low-density lipoprotein.

the group averages for each time point were similar for both product and placebo across the 20 -week study.

\section{Fasting blood glucose}

Subjects were examined for fasting blood glucose levels at each visit (Figure 5). There was no significant change in fasting glucose over the course of the 20 -week study. However, from week 8 and onward, the fasting blood glucose levels for the PTE group remained below the levels seen in the placebo group. This was seen for both sexes.

\section{Discussion}

Preserving good metabolic and cardiovascular health is important, and is related to many dietary and lifestyle factors,

Table 2 Systolic and diastolic blood pressure during the study

\begin{tabular}{|c|c|c|c|c|c|c|}
\hline & \multicolumn{2}{|l|}{ Placebo } & \multicolumn{2}{|c|}{ Puer tea extract } & \multicolumn{2}{|l|}{$P$-value } \\
\hline & Systolic & Diastolic & Systolic & Diastolic & Systolic & Diastolic \\
\hline \multicolumn{7}{|l|}{ Females } \\
\hline Baseline & $129 \pm 4.7$ & $82 \pm 2.3$ & $120 \pm 2.8$ & $75 \pm 1.9$ & 0.110 & 0.018 \\
\hline 4 weeks & $130 \pm 5.2$ & $80 \pm 2.0$ & $119 \pm 2.8$ & $75 \pm 1.9$ & 0.080 & 0.055 \\
\hline 8 weeks & $123 \pm 3.8$ & $80 \pm 1.6$ & $118 \pm 3.0$ & $76 \pm 1.8$ & 0.291 & 0.086 \\
\hline 12 weeks & $128 \pm 3.3$ & $82 \pm 2.3$ & $123 \pm 3.2$ & $76 \pm 1.9$ & 0.245 & 0.027 \\
\hline 16 weeks & $129 \pm 5.0$ & $82 \pm 2.7$ & $122 \pm 5.1$ & $74 \pm 2.2$ & 0.330 & 0.028 \\
\hline 20 weeks & $125 \pm 4.2$ & $8 I \pm 2.1$ & $121 \pm 3.7$ & $76 \pm 1.9$ & 0.404 & 0.072 \\
\hline \multicolumn{7}{|l|}{ Males } \\
\hline Baseline & $134 \pm 4.9$ & $84 \pm 2.0$ & $128 \pm 4.2$ & $82 \pm 1.5$ & 0.334 & 0.308 \\
\hline 4 weeks & $137 \pm 6.0$ & $85 \pm 2.1$ & $131 \pm 6.9$ & $81 \pm 2.7$ & 0.472 & 0.304 \\
\hline 8 weeks & $143 \pm 8 . \mid$ & $83 \pm 3.9$ & $135 \pm 7.9$ & $84 \pm 3.0$ & 0.506 & 0.842 \\
\hline 12 weeks & $138 \pm 9.0$ & $86 \pm 2.8$ & $132 \pm 5.9$ & $82 \pm 2.0$ & 0.598 & 0.280 \\
\hline 16 weeks & $132 \pm 7.2$ & $85 \pm 2.3$ & $134 \pm 8.8$ & $83 \pm 2.1$ & 0.880 & 0.544 \\
\hline 20 weeks & $134 \pm 6.2$ & $87 \pm 3.2$ & $125 \pm 3.3$ & $81 \pm 1.2$ & 0.212 & 0.121 \\
\hline
\end{tabular}

Notes: Data presented as mean \pm standard deviation unless otherwise stated. 

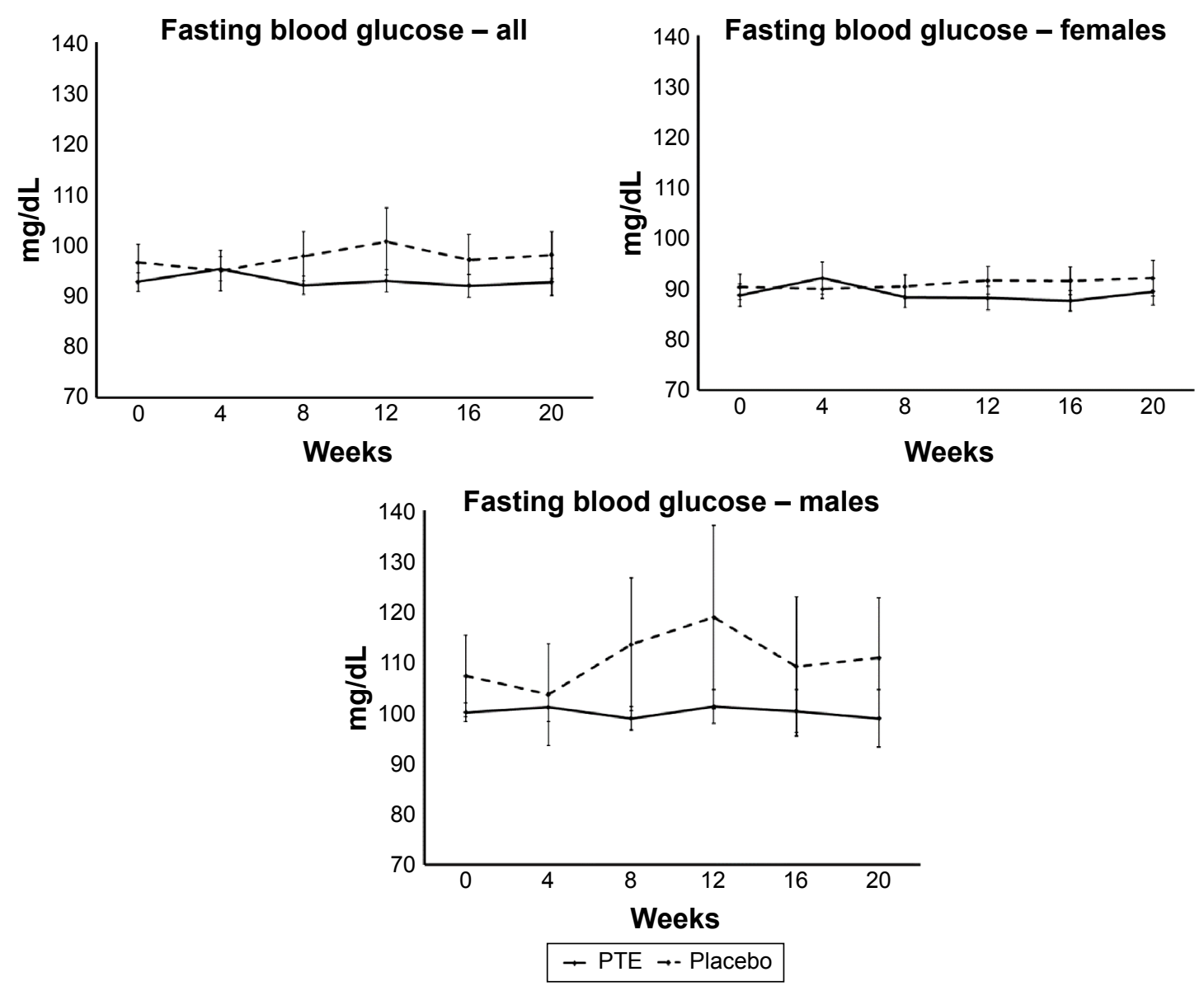

Figure 5 Fasting blood glucose levels.

Notes: Shown as group averages \pm standard error of mean for the Puer tea extract (PTE) group (solid lines) and the placebo group (dashed lines). There were no significant differences between the PTE and placebo results during the study; however, the fasting blood glucose levels remained lower in the PTE group than the placebo group from week 8 throughout the remainder of the 20 -week study.

including maintenance of healthy body weight and healthy levels of blood lipids. Obesity is a critical worldwide health concern, a major risk factor for many high-profile chronic diseases, and is of increasing global concern and impact on health-service systems. Lifestyle options, such as the daily consumption of health-promoting beverages, are practical tools to help sustain a healthy cardiovascular system, with personal and societal benefits.

Tea consumption represents a simple choice to substitute for other beverages on a daily basis, and is associated with decreased mortality, as well as specific decrease in mortality from cardiovascular and cancerous illnesses. ${ }^{34}$ Tea polyphenols have neuroprotective effects beyond their antioxidant properties, and it has been suggested that tea consumption should be considered a lifestyle choice in the prevention of neurodegenerative disorders. ${ }^{35}$ The different content of tea polyphenols in various types of tea is associated with different biological effects, and specifically Puer tea has been associated with downregulation of enzymes involved in fat storage. ${ }^{36}$
Previous clinical studies on Puer tea have been performed in Asia, where the genetic predisposition to obesity and hyperlipidemia, as well as diet and lifestyle, are distinctly different from Western regions, including Europe and North America. The data reported here are to the best of our knowledge the result of the first controlled trial on Puer tea performed outside Asia.

In line with previously published evidence, we observed a reduction in body fat in the group consuming PTE. It is important to note that the fat loss and improved blood lipids happened with no diet restrictions. Regional analysis of the DEXA data showed that the fat loss was seen for multiple regions, including the arms, legs, trunk, and the gynoid region. This is particularly interesting, since abdominal fat is associated with increased cardiovascular risk.

Lipoproteins are important and necessary for the transport of lipid nutrients and hormones in the bloodstream. However, elevated levels are associated with increased cardiovascular risk. Previous studies in animals that were 
fed a high-fat diet showed that Puer tea consumption helped maintain healthy cholesterol levels. ${ }^{12,25-30}$ Two previous clinical trials were performed in Asia, and confirmed reduced cholesterol in people consuming Puer tea. ${ }^{32,33}$ The aqueous extract of Puer tea has been shown to have specific effects on enzymes involved in lipid metabolism, and acts as a competitive inhibitor for HMGCR and pancreatic lipase and as a noncompetitive inhibitor for Lp-PLA $2 .{ }^{37}$ In the study reported here, we observed only minor changes to cholesterol and HDLs. In contrast, we saw clinically important reduction in triglycerides. Both the PTE and placebo groups had baseline levels well above $150 \mathrm{mg} / \mathrm{dL}$, but the PTE group showed a reduction within 8 weeks, where the group average was within the normal range at 8 weeks and remained within the normal range throughout the remainder of the 20-week study. This suggests that PTE may help cardiovascular health, particularly in the hypertriglyceridemic waist phenotype, which is one of the most predictive of a cardiovascular disease risk. ${ }^{38-41}$

Despite previous evidence from a rodent study that Puer tea consumption helps manage blood glucose, ${ }^{27}$ we did not see a change in fasting blood glucose in the study reported here. The data from this study suggest that daily consumption of PTE supports improved cardiovascular health in several ways, including weight management through loss of body fat and beneficial changes to blood lipids, specifically with respect to reduced triglycerides and very low-density lipoprotein. One limitation of the study reported here is that it was conducted in a hyperlipidemic population but not hyperglycemic. It is highly recommended that further studies be performed. These should aim at documenting reduction of body fat in people who are not hyperlipidemic, and should also examine the effects of consuming Puer tea in conjunction with a weight-loss program, improved glucose tolerance, and overall metabolic health through regulation of adipokines.

\section{Acknowledgments}

This study was conducted at NIS Labs, an independent contract research laboratory specializing in natural-product research. The study was sponsored by Tasly Pharmaceuticals USA.

\section{Disclosure}

GSJ and JLB are employees of NIS Labs, an independent contract research organization specializing in natural-product research, and have no competing financial interest in the subject matter. YH, ZXG, and HS are employees of Tasly Pharmaceuticals, the sponsor of this study.

\section{References}

1. World Health Organization. Obesity and overweight. 2015. Available from: http://www.who.int/mediacentre/factsheets/fs311/en. Accessed December 6, 2015.

2. Cefalu WT, Bray GA, Home PD, et al. Advances in the science, treatment, and prevention of the disease of obesity: reflections from a Diabetes Care editors' expert forum. Diabetes Care. 2015;38(8):1567-1582.

3. Wang ZJ, Zhou YJ, Galper BZ, Gao F, Yeh RW, Mauri L. Association of body mass index with mortality and cardiovascular events for patients with coronary artery disease: a systematic review and meta-analysis. Heart. 2015;101(20):1631-1638.

4. Kent BD, McNicholas WT, Ryan S. Insulin resistance, glucose intolerance and diabetes mellitus in obstructive sleep apnoea. $J$ Thorac Dis. 2015;7(8):1343-1357.

5. Scotece M, Mobasheri A. Leptin in osteoarthritis: focus on articular cartilage and chondrocytes. Life Sci. 2015;140:75-78.

6. Marengo A, Rosso C, Bugianesi E. Liver cancer: connections with obesity, fatty liver, and cirrhosis. Annu Rev Med. Epub 2015 Oct 14.

7. Orecchioni S, Reggiani F, Talarico G, Bertolini F. Mechanisms of obesity in the development of breast cancer. Discov Med. 2015;20(109): 121-128.

8. Allott EH, Hursting SD. Obesity and cancer: mechanistic insights from transdisciplinary studies. Endocr Relat Cancer. 2015;22(6): R365-R386.

9. Calle EE, Thun MJ, Petrelli JM, Rodriguez C, Heath CW Jr. Body-mass index and mortality in a prospective cohort of U.S. adults. $N$ Engl J Med. 1999;341(15):1097-1105.

10. Eckel RH, Krauss RM. American Heart Association call to action: obesity as a major risk factor for coronary heart disease. Circulation. 1998;97(21):2099-2100.

11. Rueda-Clausen CF, Ogunleye AA, Sharma AM. Health benefits of longterm weight-loss maintenance. Annu Rev Nutr. 2015;35:475-516.

12. Huang HC, Lin JK. Pu-erh tea, green tea, and black tea suppresses hyperlipidemia, hyperleptinemia and fatty acid synthase through activating AMPK in rats fed a high-fructose diet. Food Funct. 2012;3(2): $170-177$.

13. Way TD, Lin HY, Kuo DH, et al. Pu-erh tea attenuates hyperlipogenesis and induces hepatoma cells growth arrest through activating AMPactivated protein kinase (AMPK) in human HepG2 cells. J Agric Food Chem. 2009;57(12):5257-5264.

14. Duh PD, Yen GC, Yen WJ, Wang BS, Chang LW. Effects of Pu-erh tea on oxidative damage and nitric oxide scavenging. J Agric Food Chem 2004;52(26):8169-8176.

15. Ku KM, Kim J, Park HJ, Liu KH, Lee CH. Application of metabolomics in the analysis of manufacturing type of Pu-erh tea and composition changes with different postfermentation year. J Agric Food Chem. 2010; 58(1):345-352.

16. Tian LW, Tao MK, Xu M, et al. Carboxymethyl- and carboxyl-catechins from ripe Pu-er tea. J Agric Food Chem. 2014;62(50):12229-12234.

17. Zhao LF, Zhou HJ, An WJ. [Study on health promoting effect of Yunnan Pu-erh tea]. Shipin Yanjiu Yu Kaifa. 2005;26(2):114-118. Chinese.

18. Du WH, Peng SM, Liu ZH, Shi L, Tan LF, Zou XQ. Hypoglycemic effect of the water extract of Pu-erh tea. J Agric Food Chem. 2012; 60(40):10126-10132.

19. Huang Q, Chen S, Chen H, et al. Studies on the bioactivity of aqueous extract of Pu-erh tea and its fractions: in vitro antioxidant activity and $\alpha$-glycosidase inhibitory property, and their effect on postprandial hyperglycemia in diabetic mice. Food Chem Toxicol. 2013;53:75-83.

20. Yan SJ, Wang L, Li Z, et al. Inhibition of advanced glycation end product formation by $\mathrm{Pu}$-erh tea ameliorates progression of experimental diabetic nephropathy. J Agric Food Chem. 2012;60(16):4102-4110.

21. Cao ZH, Gu DH, Lin QY, et al. Effect of Pu-erh tea on body fat and lipid profiles in rats with diet-induced obesity. Phytother Res. 2011; 25(2):234-238.

22. Yamashita Y, Wang L, Wang L, Tanaka Y, Zhang T, Ashida H. Oolong, black and Pu-erh tea suppresses adiposity in mice via activation of AMP-activated protein kinase. Food Funct. 2014;5(10):2420-2429. 
23. Gong J, Peng C, Chen T, Gao B, Zhou H. Effects of theabrownin from $\mathrm{Pu}$-erh tea on the metabolism of serum lipids in rats: mechanism of action. J Food Sci. 2010;75(6):H182-H189.

24. Hou Y, Shao W, Xiao R, et al. Pu-erh tea aqueous extracts lower atherosclerotic risk factors in a rat hyperlipidemia model. Exp Gerontol. 2009;44(6-7):434-439.

25. Kuo KL, Weng MS, Chiang CT, Tsai YJ, Lin-Shiau SY, Lin JK. Comparative studies on the hypolipidemic and growth suppressive effects of oolong, black, Pu-erh, and green tea leaves in rats. J Agric Food Chem. 2005;53(2):480-489.

26. Wang Q, Gong J, Chisti Y, Sirisansaneeyakul S. Fungal isolates from a $\mathrm{Pu}$-erh type tea fermentation and their ability to convert tea polyphenols to theabrownins. J Food Sci. 2015;80(4):M809-M817.

27. Deng YT, Lin-Shiau SY, Shyur LF, Lin JK. Pu-erh tea polysaccharides decrease blood sugar by inhibition of $\alpha$-glucosidase activity in vitro and in mice. Food Funct. 2015;6(5):1539-1546.

28. Yang DJ, Hwang LS. Study on the conversion of three natural statins from lactone forms to their corresponding hydroxy acid forms and their determination in Pu-Erh tea. J Chromatogr A. 2006;1119(1-2):277-284.

29. Jeng KC, Chen CS, Fang YP, Hou RC, Chen YS. Effect of microbial fermentation on content of statin, GABA, and polyphenols in Pu-erh tea. J Agric Food Chem. 2007;55(21):8787-8792.

30. Hou CW, Jeng KC, Chen YS. Enhancement of fermentation process in Pu-erh tea by tea-leaf extract. J Food Sci. 2010;75(1):H44-H48.

31. Zhao ZJ, Pan YZ, Liu QJ, Li XH. Exposure assessment of lovastatin in Pu-erh tea. Int J Food Microbiol. 2013;164(1):26-31.

32. Fujita H, Yamagami T. Antihypercholesterolemic effect of Chinese black tea extract in human subjects with borderline hypercholesterolemia. Nutr Res. 2008;28(7):450-456.

33. Kubota K, Sumi S, Tojo H, et al. Improvements of mean body mass index and body weight in preobese and overweight Japanese adults with black Chinese tea (Pu-erh) water extract. Nutr Res. 2011;31(6):421-428.
34. Tang J, Zheng JS, Fang L, Jin Y, Cai W, Li D. Tea consumption and mortality of all cancers, CVD and all causes: a meta-analysis of eighteen prospective cohort studies. Br J Nutr. 2015;114(5):673-683.

35. Caruana M, Vassallo N. Tea polyphenols in Parkinson's disease. Adv Exp Med Biol. 2015;863:117-137.

36. Ding Y, Zou X, Jiang X, et al. Pu-erh tea down-regulates sterol regulatory element-binding protein and stearyol[sic]-CoA desaturase to reduce fat storage in Caenorhaditis elegans. PLoS One. 2015;10(2):e0113815.

37. Zeng L, Yan J, Luo L, Zhang D. Effects of Pu-erh tea aqueous extract (PTAE) on blood lipid metabolism enzymes. Food Funct. 2015;6(6): 2008-2016.

38. Lemieux I, Pascot A, Couillard C, et al. Hypertriglyceridemic waist: a marker of the atherogenic metabolic triad (hyperinsulinemia, hyperapolipoprotein B, small, dense LDL) in men? Circulation. 2000;102(2):179-184.

39. Czernichow S, Bruckert E, Bertrais S, Galan P, Hercberg S, Oppert JM. Hypertriglyceridemic waist and 7.5-year prospective risk of cardiovascular disease in asymptomatic middle-aged men. Int $J$ Obes (Lond). 2007;31(5):791-796.

40. Tankó LB, Bagger YZ, Qin G, Alexandersen P, Larsen PJ, Christiansen C. Enlarged waist combined with elevated triglycerides is a strong predictor of accelerated atherogenesis and related cardiovascular mortality in postmenopausal women. Circulation. 2005;111(15):1883-1890.

41. Nazare JA, Smith J, Borel AL, et al. Usefulness of measuring both body mass index and waist circumference for the estimation of visceral adiposity and related cardiometabolic risk profile (from the INSPIRE ME IAA study). Am J Cardiol. 2015;115(3):307-315.
Clinical Interventions in Aging

\section{Publish your work in this journal}

Clinical Interventions in Aging is an international, peer-reviewed journal focusing on evidence-based reports on the value or lack thereof of treatments intended to prevent or delay the onset of maladaptive correlates of aging in human beings. This journal is indexed on PubMed Central, MedLine,

\section{Dovepress}

CAS, Scopus and the Elsevier Bibliographic databases. The manuscript management system is completely online and includes a very quick and fair peer-review system, which is all easy to use. Visit http://www.dovepress. com/testimonials.php to read real quotes from published authors. 